\title{
Skeletal maturity assessment using mandibular canine calcification stages
}

\author{
Vildana Džemidžić́ ${ }^{1}$, Alisa Tiro ${ }^{1}$, Amila Zukanović́ ${ }^{2}$, Ismeta Redžić ${ }^{3}$, Enita Nakaš ${ }^{1}$
}

${ }^{1}$ Department of Orthodontics, School of Dental Medicine, University of Sarajevo Bosnia and Herzegovina, ${ }^{2}$ Department of Preventive and Pediatric Dentistry School of Dental Medicine, University of Sarajevo, Bosnia and Herzegovina ${ }^{3}$ Faculty of Medicine, University of Sarajevo, Bosnia and Herzegovina

Correspondence: vdzemidzic@hotmail.com

Tel.: + 38733407830

Fax.: + 38733443395

Received: 6 July 2016

Accepted: 30 September 2016

Key words: Orthodontics - Mandibular canine - Calcification stage - Cervical vertebrae - Skeletal maturation.
Objective. The aims of this study were: to investigate the relationship between mandibular canine calcification stages and skeletal maturity; and to evaluate whether the mandibular canine calcification stages may be used as a reliable diagnostic tool for skeletal maturity assessment. Materials and methods. This study included 151 subjects: 81 females and 70 males, with ages ranging from 9 to 16 years (mean age: $12.29 \pm 1.86$ years). The inclusion criteria for subjects were as follows: age between 9 and 16 years; good general health without any hormonal, nutritional, growth or dental development problems. Subjects who were undergoing or had previously received orthodontic treatment were not included in this study. The calcification stages of the left permanent mandibular canine were assessed according to the method of Demirjian, on panoramic radiographs. Assessment of skeletal maturity was carried out using the cervical vertebral maturation index (CVMI), as proposed by the Hassel-Farman method, on lateral cephalograms. The correlation between the calcification stages of mandibular canine and skeletal maturity was estimated separately for male and female subjects. Results. Correlation coefficients between calcification stages of mandibular canine and skeletal maturity were 0.895 for male and 0.701 for female subjects. Conclusions. A significant correlation was found between the calcification stages of the mandibular canine and skeletal maturity. The calcification stages of the mandibular canine show a satisfactory diagnostic performance only for assessment of pre-pubertal growth phase.

\section{Introduction}

Timing plays a crucial role in the outcomes of treatment for skeletal disharmonies in growing patients. Successful treatment of skeletal disharmonies may be achieved by growth modification in patients that have a certain amount of growth remaining (1-3). There are wide individual variations in the timing, duration and velocity of growth, and therefore we need to assess individual development stages (4). Skeletal maturity may be assessed by means of several more or less reliable parameters: chronological age (5), increases in body height and weight (6), secondary sexual characteristics (6), dental maturity (calcification stages) (7), hand and wrist maturational stages (8), cervical vertebral maturation $(3,9)$ and biochemical markers $(10,11)$.

Dental development is one of the important indicators of skeletal maturity. There are two possibilities for assessment of skeletal maturity. One of them is tooth erup- 
tion, which is not a reliable parameter of skeletal maturity. Franchi et al. (12) showed a poor correlation between the phases of dentition and skeletal maturity. In addition to tooth eruption, dental maturity is estimated through the stages of calcification, and this is a much more reliable parameter for assessment of skeletal maturity (13-16). For all these reasons, dental maturity is recommended as a clinically useful diagnostic tool for assessment of skeletal maturity. Assessment of skeletal maturity, based on the stages of tooth calcification, has certain advantages, as it allows assessment based on panoramic radiographs that are routinely used for diagnosis, and assessment may be carried out by analysing retroalveolar radiographs. Stages of teeth development are associated with growth and development, which means that dental maturity may be used as a parameter for assessment of skeletal maturity, as has been shown by numerous studies (13-16). Some authors noted that the developmental stages of certain teeth, for example canines $(17,18)$ and second molars (19), show a high correlation with skeletal maturity.

The aims of this study were: (1) to investigate the relationship between mandibular canine calcification stages and skeletal maturity; and (2) to evaluate whether the mandibular canine calcification stages may be used as a reliable diagnostic tool for skeletal maturity assessment.

\section{Subjects and methods}

This study included 151 subjects: 81 females and 70 males, with ages ranging from 9 to 16 years (mean age: $12.29 \pm 1.86$ years). The mean age of the males was $12.05( \pm 1.83)$, and for females $12.31( \pm 1.89)$ years. Chronological age was recorded according to the date of birth, confirmed by the parents. The inclusion criteria for subjects were as follows: age between 9 and 16 years, and good general health without any hormonal, nutritional, growth or dental development problems. Subjects who were undergoing or had previously received orthodontic treatment were not included in this study. Assessment of mandibular canine calcification and cervical vertebral maturation stages were performed using good quality panoramic radiographs and lateral cephalograms. All analyses were conducted by an experienced orthodontist.

\section{Assessment of mandibular canine calcification stages}

The calcification stages of the left permanent mandibular canine were assessed according to the method of Demirjian et al. (7) (stages E to $H$ ), on panoramic radiographs. These stages are defined as:

- Stage E - The walls of the pulp chamber form straight lines, the continuity of which is broken by the presence of the pulp horn, which is larger than in the previous stage and the root length is less than the crown height.

- Stage F - The walls of the pulp chamber form an isosceles triangle, with apex ending in a funnel shape, and the root length is equal to or greater than the crown height.

- Stage G - The walls of the root canal are parallel and its apical end is still partially open.

- Stage $\mathrm{H}$ - the apical end of the root canal is completely closed, and the periodontal membrane has a uniform width around the root and the apex.

\section{Assessment of individual skeletal maturity}

Assessment of skeletal maturity was carried out using the cervical vertebral maturation index (CVMI), as proposed by the HasselFarman method (9), on lateral cephalograms. This method requires that the second, third and fourth cervical vertebrae 


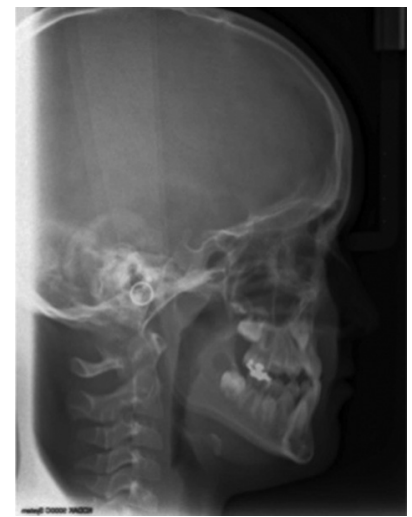

a) CVMI 1 - Initiation

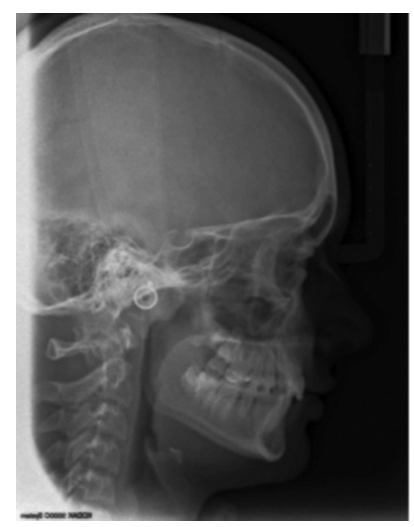

d) CVMI 4 - Deceleration

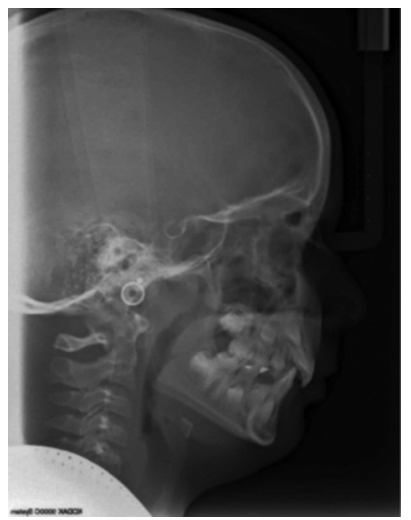

b) CVMI 2 - Acceleration

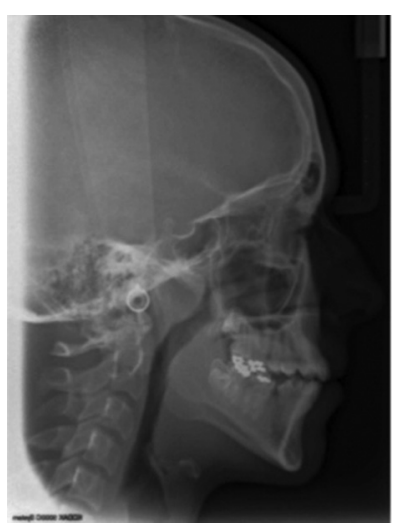

e) CVMI 5 - Maturation

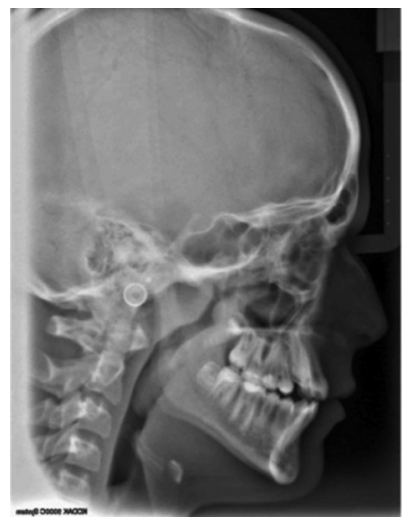

c) CVMI 3 - Transition

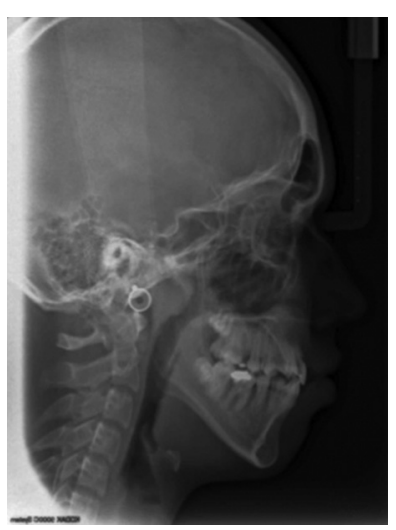

f) CVMI 6 - Completion

Figure 1 The stages of cervical vertebral maturation according to the Hassel-Farman method.

(C2, C3 and C4) are analysed according to their shape and classified into one of the six stages, which are defined as follows:

- CVMI 1 Initiation: The lower borders of the second, third and fourth cervical vertebrae (C2, C3 and C4) are flat. The bodies of $\mathrm{C} 3$ and $\mathrm{C} 4$ are trapezoid in shape and the superior vertebral borders are tapered from posterior to anterior (Figure 1a).

- CVMI 2 Acceleration: Concavities develop on the inferior borders of C2 and C3, and the inferior border of $\mathrm{C} 4$ is flat. The bodies of $\mathrm{C} 3$ and $\mathrm{C} 4$ are nearly rectangular in shape (Figure 1b).

- CVMI 3 Transition: Distinct concavities develop on the inferior borders of $\mathrm{C} 2$ and C3, and concavity begins to develop on the inferior border of $\mathrm{C} 4$. The bodies of $\mathrm{C} 3$ and $\mathrm{C} 4$ are rectangular (Figure 1c).
- CVMI 4 Deceleration: Distinct concavities are seen on the inferior borders of $\mathrm{C} 2, \mathrm{C} 3$ and $\mathrm{C} 4$. The vertebral bodies are becoming more square in shape (Figure 1d).

- CVMI 5 Maturation: More accentuated concavities are seen on the inferior borders of $\mathrm{C} 2, \mathrm{C} 3$ and C4. The bodies of $\mathrm{C} 3$ and C4 are nearly square in shape (Figure 1e).

- CVMI 6 Completion: Deep concavities are seen on the inferior borders of $\mathrm{C} 2$, $\mathrm{C} 3$ and C4. The bodies of C3 and C4 are square or greater in vertical dimension than in horizontal dimension (Figure 1f).

\section{Ethics statement}

The study was approved by the Ethical Committee of the School of Dental Medicine, University of Sarajevo. 


\section{Statistical analysis}

SPSS software, version 20 was used to perform the statistical analyses. The frequency and the percentage distribution of the CVMI stages and mandibular canine calcification stages were calculated separately for male and female subjects. Correlation between CVMI and the mandibular canine calcification stage was presented through Spearman's correlation coefficient. The level of significance was defined as $\mathrm{p}<0.001$.

\section{Results}

The distributions of the different calcification stages of the mandibular canine for male subjects are shown in Table 1.
Calcification stages E (42.9\%) and F (57.1\%) of the mandibular canine were found at stage 1 of CVMI. At stage 2 of CVMI $82.6 \%$ of subjects were in calcification stage $\mathrm{F}$ and $17.4 \%$ of subjects were in stage G, while at stage 3 of CVMI only $11.1 \%$ of subjects were in stage $\mathrm{F}, 66.7 \%$ were in stage $\mathrm{G}$ and $22.2 \%$ were in stage $\mathrm{H}$. At stage 4 of CVMI $17.4 \%$ of subjects were in stage $\mathrm{G}$ and $82.6 \%$ of subjects were in stage $\mathrm{H}$. All subjects in stage 5 of CVMI were in calcification stage $\mathrm{H}$ of the mandibular canine. However, none of the male subjects was at stage 6 of CVMI. Spearman's correlation was used to assess the correlation between CVMI stages and mandibular canine calcification stages.

Table 1 Contingency table showing distributions between the different calcification stages of mandibular canine and CVMI stages for male subjects

\begin{tabular}{|c|c|c|c|c|c|}
\hline \multirow{3}{*}{ CVMI } & \multicolumn{4}{|c|}{ Stages of calcification of mandibular canine } & \multirow{2}{*}{ Total } \\
\hline & E & $\mathrm{F}$ & G & $\mathrm{H}$ & \\
\hline & n (\%) & $\mathrm{n}(\%)$ & n (\%) & $\mathrm{n}(\%)$ & $\mathrm{n}(\%)$ \\
\hline 1 & $3(42.9)$ & $4(57.1)$ & - & - & $7(100)$ \\
\hline 2 & - & $19(82.6)$ & $4(17.4)$ & - & $23(100)$ \\
\hline 3 & - & $1(11.1)$ & $6(66.7)$ & $2(22.2)$ & $9(100)$ \\
\hline 4 & - & - & $4(17.4)$ & $19(82.6)$ & $23(100)$ \\
\hline 5 & - & - & - & $8(100)$ & $8(100)$ \\
\hline Total & $3(4.3)$ & $24(34.3)$ & $14(20.0)$ & $29(41.4)$ & $70(100)$ \\
\hline
\end{tabular}

CVMI=Cervical Vertebral Maturation Index (Spearman's rho correlation coefficient between calcification stages of mandibular canine and CVMI is $0.895, \mathrm{p}<0.001$ ).

Table 2 Contingency table showing distributions between the different calcification stages of mandibular canine and CVMI stages for female subjects

\begin{tabular}{|c|c|c|c|c|}
\hline \multirow{2}{*}{ CVMI } & \multicolumn{3}{|c|}{ Stages of calcification of mandibular canine } & \multirow{2}{*}{ Total } \\
\hline & $\mathrm{F}$ & G & $\mathrm{H}$ & \\
\hline & n (\%) & n (\%) & n (\%) & n (\%) \\
\hline 1 & $1(100)$ & 0 & 0 & $1(100)$ \\
\hline 2 & $8(66.7)$ & $4(33.3)$ & 0 & $12(100)$ \\
\hline 3 & 0 & $7(46.7)$ & $8(53.3)$ & $15(100)$ \\
\hline 4 & 0 & $6(31.6)$ & $13(68.4)$ & $19(100)$ \\
\hline 5 & 0 & $1(3.7)$ & $26(96.3)$ & $27(100)$ \\
\hline 6 & 0 & 0 & $7(100)$ & $7(100)$ \\
\hline Total & $9(11.1)$ & $18(22.2)$ & $54(66.7)$ & $81(100)$ \\
\hline
\end{tabular}

CVMI=Cervical Vertebral Maturation Index (Spearman's rho correlation coefficient between calcification stages of mandibular canine and CVMI is $0.701 ; \mathrm{p}<0.001)$. 
Among the female subjects calcification stages F, G and $\mathrm{H}$ of the mandibular canine were found. Only one subject was at stage $1(100 \%)$ of CVMI, with calcification stage $\mathrm{F}$ of the mandibular canine. At stage 2 of CVMI $66.7 \%$ of subjects were in calcification stage $\mathrm{F}$, and $33.3 \%$ of subjects were in stage G. At stages 3, 4 and 5 of CVMI, the subjects were in calcification stages $G$ and $\mathrm{H}$ of the mandibular canine. At stage 6 of CVMI all subjects were in calcification stage $\mathrm{H}$ of the mandibular canine. The correlation between CVMI and the calcification stage of the mandibular canine for female subjects was analysed by Spearman's correlation coefficient.

\section{Discussion}

In the present study, tooth calcification stages were preferred rather than eruption because tooth formation is proposed as a more reliable parameter. The method according to Demirjian was used, because this method is based on shape criteria and the proportion of root length, using the relative values of crown height rather than the absolute length (7). Therefore, foreshortened or elongated projections of developing teeth will not affect the reliability of assessment (20).

Among all the tested teeth, mandibular canines showed a significant association with the stages of development (17-21). In addition, hypodontia of permanent canine is extremely rare, and the prevalence ranges from 0.18 to $0.29 \%$ (22). Also, mandibular permanent canine rarely show morphological variations in their development (23).

Assessment of skeletal maturity was carried out using the cervical vertebral maturation index (CVMI), as the proposed HasselFarman method, on lateral cephalograms (9), is a routine diagnostic radiograph for orthodontic treatment. Studies have found that CVMI is a reliable method for skeletal maturity assessment $(9,13,15,19,21)$.
Some studies report high correlations between calcification stages of teeth (dental maturity) and skeletal maturity indicators (13-16). Some authors investigated correlations between calcification stages of some teeth (canines, second and third molars), and they found that the calcification stage of mandibular canine showed a significant correlation with skeletal maturity $(17,18$, 21). In this study, the correlation coefficients between calcification stages of mandibular canine and skeletal maturity were 0.895 for male and 0.701 for female subjects, and all correlation coefficients were statistically significant, at $\mathrm{p}<0.001$. These results indicate that the calcification stages of mandibular canine are a reliable parameter for skeletal maturity assessment.

CVMI stage 2 indicates the beginning of the pubertal growth spurt (9). In our study, calcification stage $\mathrm{F}$ of the mandibular $\mathrm{Ca}$ nine coincided well with the CVMI stage 2, for both sexes (Table 1 and 2). Mittal et al. (15) and Goyal et al. (21) reported similar results in their studies. This means that calcification stage $\mathrm{F}$ of the mandibular canine could be used for assessing the pre-pubertal growth phase. According to the Hassel and Farman method, at CVMI stage 3 growth is still accelerating toward peak velocity; while at CVMI stage 4 adolescent growth begins to decelerate (9). Calcification stage $G$ of the mandibular canine coincided well with CVMI stage 3 , and stage $\mathrm{H}$ with CVMI stage 4 for the male subjects in our study. However, for female subjects calcification stage $\mathrm{H}$ of the mandibular canine showed the highest percentage distribution at CVMI stages 3 and 4 . For both genders, canine root formation was complete (stage $\mathrm{H}$ ) in the majority of subjects at CVMI stages 5 and 6 . These results are consistent with the results of other similar studies $(15,16,21)$.

In this study, dental maturation of the mandibular canine occurred earlier in female subjects than in male subjects, which 
was in accordance with a previous study (21). In contrast, the results of the studies by Chertcow (13) and Uysal et al. (17) showed advanced dental maturation in male subjects. These results indicate that the calcification stages of mandibular canines may be considered as a reliable parameter to assess pre-pubertal growth phases.

\section{Conclusions}

A significant correlation between the calcification stages of the mandibular canine and skeletal maturity was found. The calcification stages of the mandibular canine show satisfactory diagnostic performance only for assessment of pre-pubertal growth phases.

\section{What is already known on this topic}

Successful treatment of the skeletal disharmonies may be achieved by growth modification, in patients that have a certain amount of growth remaining. There are wide individual variations in the beginning, amount and duration of pubertal growth acceleration, and therefore an assessment of individual development stages is needed. Individual skeletal maturity may be assessed by means of several, more or less reliable parameters.

\section{What this study adds}

The calcification stages of mandibular canine show satisfactory diagnostic performance for assessment of pre-pubertal growth phase.

Authors' contributions: Conception and design: VDZ; Acquisition, analysis and interpretation of data: VDZ, AT, AZ and IR; Drafting the article: VDZ; Revising it critically for important intellectual content: EN; Approved final version of the manuscript: VDZ, AT and EN.

Conflict of interest: The authors declare that they have no conflict of interest.

\section{References}

1. Malmgren O, Ömblus J, Hägg U, Pancherz H. Treatment with an orthopedic appliance system in relation to treatment intensity and growth periods. A study of initial effect. Am J Orthod Dentofacial Orthop. 1987;91(2):143-51.
2. Faltin K, Faltin R, Baccetti T, Franchi L, Ghiozzi B, McNamara JA. Long-term Effectiveness and Treatment Timing for Bionator Therapy. Angle Orthod. 2003;73(3):221-30.

3. Baccetti T, Franchi L, McNamara JA. The Cervical Vertebral Maturation (CVM) Method for the Assessment of Optimal Treatment Timing in Dentofacial Orthopedics. Semin Orthod. 2005;11:11929.

4. Silventoinen K, Haukka J, Dunkel L, Tynelius P, Rasmussen F. Genetics of Pubertal Timing and Its Associations With Relative Weight in Childhood and Adult Height: The Swedish Young Male Twins Study. Pediatrics. 2008;121(4):e885-91.

5. Alkhal HA, Wong RWK, Rabie ABM. Corelation between Chronological Age, Cervical Vertebral Maturation and Fishman's Skeletal Maturity Indicators in Southern Chinese. Angle Orthod. 2008;78(4):591-6.

6. Hägg U, Taranger J. Maturation indicators and the pubertal growth spurt. Am J Orthod Dentofacial Orthop. 1982;82(4):299-309.

7. Demirjian A, Goldstein H, Tanner JM. A New System of Dental Age Assessment. Human Biology. 1973;45(2):211-27.

8. Fishman LS. Radiographic Evaluation of Skeletal Maturation. A Clinically Oriented Method Based on Hand-Wrist Films. Angle Orthod. 1982;52(2):88-112.

9. Hassel B, Farman AG. Skeletal maturation evaluation using cervical vertebrae. Am J Orthod Dentofac Orthop. 1995;107(1):58-66.

10. Perinetti G, Baccetti T, Contardo L, Di Lenarda R. Gingival crevicular fluid alkaline phosphatase activity as a non-invasive biomarker of skeletal maturation. Orthod Craniofac Res. 2011;14(1):44-50.

11. Perinetti G, Contardo L. Gingival crevicular fluid alkaline phosphatase activity in relation to pubertal growthz spurt and dental maturation: A multiple regression study. South Eur J Orthod Dentofac Res. 2016;3(1):6-11.

12. Franchi L, Baccetti T, Toffol LD, Polimeni A, Cozza P. Phases of the dentition for assessment of skeletal maturity: A diagnostic performance study. Am J Orthod Dentofacial Orthop. 2008;133(3):395-400.

13. Uysal T, Sari Z, Ramoglu SI, Basciftci FA. Relationship Between Dental and Skeletal Maturity in Turkish Subjects. Angle Orthod. 2004;74(5):65764.

14. Chen J, Hu H, Guo J, Liu Z, Liu R, Li F, Zou S. Correlation between dental maturity and cervical vertebral maturity. Oral Surg Oral Med Oral Pathol Oral Radiol Endod. 2010;110(6):777-83. 
15. Mittal SK, Singla A, Virdi MS, Sharma R, Mittal B. Co-Relation Between Determination Of Skeletal Maturation Using Cervical Vertebrae And Dental Calcification Stages. The Internet Journal of Forensic Science. 2009;4(2) [Last accessed on 2016 May 17]. Available from: https://134 www.ispnb.com/IJFS/4/2/5855.

16. Perinetti G, Contardo L, Gabrieli P, Baccetti T, Di Lenarda R. Diagnostic performance of dental maturity for identification of skeletal maturation phase. Eur J Orthod. 2012;34(4):487-92.

17. Chertkow $S$. Tooth mineralization as an indicator of the pubertal growth spurt. Am J Orthod Dentofacial Orthop. 1980;77(1):79-91.

18. Coutinho S, Baschang PH, Miranda F. Relationships between mandibular canine calcification stages and skeletal maturity. Am J Orthod Dentofacial Orthop. 1993;104:262-8.

19. Kumar S, Singla A, Sharma R, Virdi MS, Anupam A, Mittal B. Skeletal maturation evaluation using mandibular second molar calcification stages. Angle Orthod. 2012;82(3):501-6.

20. Krailassiri S, Anuwongnukroh N, Dechkunakorn S. Relationship Between Dental Calcification Stages and Skeletal Maturity Indicators in Thai Individuals. Angle Orthod. 2002;72(2):155-66.

21. Goyal S, Goyal S, Gugnani N. Assessment of skeletal maturity using the permanent mandibular canine calcification stages. J Orthod Res. 2014;2(1):11-6.

22. Rózsa N, Nagy K, Vajó Z, Gábris K, Soós A, Alberth $\mathrm{M}$, et al. Prevalence and distribution of permanent canine agenesis in dental paediatric and orthodontic patients in Hungary. Eur J Orthod. 2009;31(4):374-9.

23. Burić MV, Tijanić LjD, Janošević PM, Filipović GLj, Stojanović DK. Asymetry in development (mineralisation) of permanent mandibular canine roots. Acta Stomatologica Naissi. 2012;28:1191-6. 